\title{
Físic@ virtu@I: ambiente Moodle de objetos de aprendizagem de física como ferramenta auxiliar nos estudos dos alunos
}

\author{
Vítor Freire e Salvador - PUCRS - vitor.salvador@acad.pucrs.br \\ Rosana Maria Gessinger - PUCRS - rosana.gessinger@ pucrs.br \\ Maria do Carmo Baptista Lagreca - PUCRS - lagreca@ pucrs.br
}

Resumo: Este artigo apresenta o relato de uma experiência desenvolvida em uma Instituição de Ensino Superior (IES) que teve por objetivo auxiliar alunos ingressantes dos cursos de Física e de Engenharia na reconstrução de conhecimentos básicos de física. O projeto foi desenvolvido em 5 etapas: (1) desenvolvimento do ambiente Físic@ Virtu@l; (2) adaptação de oficinas para o ambiente virtual e criação de objetos de aprendizagem contemplando outros temas; (3) disponibilização do ambiente Físic@ Virtu@l para o acesso de alunos cursando disciplinas de física básica; (4) manutenção do ambiente virtual e ampliação dos objetos de aprendizagem; (5) criação e disponibilização de instrumentos para avaliar o ambiente virtual e os objetos. Evidenciou-se que o número de acessos ao ambiente indica a relevância desta iniciativa no apoio à aprendizagem.

Palavras-chave: ambiente virtual de aprendizagem; objetos de aprendizagem; aprendizagem de física

\section{Físic@virtu@l: Moodle environment of physics learning objects as a tool that helps in students' studies}

\begin{abstract}
This article presents an experience developed in a Higher Education Institution and aimed to help students of Physics and Engineering courses in the reconstruction of Physics basic knowledge. The project was developed in 5 phases: (1) development of the virtual environment called Físic@Virtu@1; (2) adaptation of workshops that used learning objects available in the Institution Learning Lab for the Físic@Virtu@1 and development of learning objects; (3) availability of the Físic@Virtu@1 for students who attended some Basic Physics discipline; (4) maintenance of the virtual environment and development of new learning objects; (5) construction and availability of tools to evaluate the virtual environment. The number of access to the virtual environment indicates the relevance of this initiative in supporting learning.
\end{abstract}

Keywords: virtual learning environment; learning objects; Physics learning

\section{Introdução}

Uma preocupação recorrente de professores universitários refere-se aos conhecimentos prévios que estudantes ingressantes no nível superior, especificamente nos cursos de ciências exatas, deveriam ter para um bom aproveitamento das disciplinas ofertadas em seus respectivos currículos. Nos cursos de graduação em Física e 
Engenharia, por exemplo, os conhecimentos construídos no ensino médio servem de base para as construções futuras e falhas nessa construção podem constituir-se no primeiro obstáculo enfrentado pelos estudantes, podendo gerar frustrações e até mesmo a evasão do curso.

Estudos têm evidenciado fragilidades no que se refere aos conhecimentos prévios manifestados pelos estudantes ao cursarem uma graduação. Gomes et al. (2005), discutem tais fragilidades, afirmando que alguns alunos de cursos de Engenharia chegam à universidade com lacunas em conteúdos relacionados à aritmética e álgebra que deveriam ter sido aprendidos nos anos finais do ensino fundamental.

A questão que se coloca é como proceder diante desse contexto e criar condições para a permanência dos estudantes na universidade. Não se pode penalizar os estudantes por eventuais falhas na sua formação no ensino médio, tampouco refazer esta etapa de ensino na graduação, pois é necessário aprofundar e ampliar os conhecimentos. É necessário, portanto, a criação de estratégias de ensino que contribuam para que os estudantes consigam acompanhar e concluir com êxito o curso de graduação escolhido.

Gomes et al. (2005) desenvolveram um projeto pedagógico chamado Cálculo Zero, que consistia na disponibilização de materiais de apoio a ingressantes do curso de Engenharia, pois perceberam que muitos deles reprovavam nas disciplinas de Cálculo devido ao desempenho muito fraco no primeiro ano, levando eventualmente à troca de curso. Quartieri et al. (2012, p. 1) desenvolveram um trabalho semelhante, reestruturando uma disciplina chamada Fundamentos da Matemática, juntamente com professores de Física e de Matemática, para "suprir dificuldades trazidas pelos alunos ingressantes nos cursos do Centro de Ciências Exatas e Tecnológicas, oriundas de uma aprendizagem pouco significativa do Ensino Médio".

Uma possível estratégia para ajudar estudantes em sua trajetória acadêmica é a utilização de repositórios de objetos de aprendizagem, applets e simulações de acesso público. Os objetos podem ajudar o professor em sua ação pedagógica e o aluno no desenvolvimento da autonomia e da capacidade de resolver problemas. Nesta perspectiva, Lagreca et al. (2012) desenvolveram objetos de aprendizagem de física, objetivando o auxílio à aprendizagem de alunos ingressantes de cursos que possuem disciplinas de física nos semestres iniciais. Nicola e Rodrigues (2011) concluíram, em seu estudo, que os objetos de aprendizagem são potencializadores para a compreensão de conteúdos de Biologia. Sobrinho et al. (2006) evidenciaram que os objetos de aprendizagem são recursos que contribuem para os processos de ensino e de aprendizagem de língua inglesa.

Diante desse contexto, este artigo tem como objetivo apresentar o relato de uma experiência realizada em uma Instituição de Ensino Superior do sul do país, que consistiu no desenvolvimento de ações com vistas a auxiliar, no processo de formação, alunos de cursos que possuem disciplinas de física nos semestres iniciais. As ações envolveram a criação do ambiente Moodle de objetos de aprendizagem de física, intitulado Físic@ virtu@l.

O artigo está organizado em quatro seções, incluindo a Introdução, na qual foram apresentadas a justificativa do estudo e o seu objetivo. Na segunda seção, Projeto Físic@ Virtu@l, abordam-se as etapas do desenvolvimento do projeto. Na terceira seção, Apresentação dos resultados, apresentam-se os resultados da avaliação desenvolvida ao longo do projeto. Na última seção, Considerações finais, retoma-se o problema inicial e apresentam-se algumas implicações do projeto.

V. $15 \mathrm{~N}^{\mathrm{o}} 1$, julho, 2017 


\section{Projeto Físic@Virtu@I}

O ProjetoFísic@Virtu@l partiu do entendimento de que a disponibilização de ambientes virtuais com objetos de aprendizagem pode contribuir para a aprendizagem dos estudantes. Um objeto de aprendizagem difere dos demais recursos didáticos por ser reutilizável e passível de ser utilizado em diferentes plataformas, por poder conter ou estar contido em outros objetos, não dependendo de outros objetos para fazer sentido e por ser descrito por metadados (Sabbatini, 2012). Portanto, um objeto é um recurso digital que pode ser usado, reutilizado e combinado com outros diferentes objetos a fim de otimizar a experiência de aprendizagem do aluno. No geral, usam-se simulações, vídeos, imagens, aplicativos, entre outros, para uma maior interatividade entre o objeto de aprendizagem e o aluno, tornando a experiência de aprender mais lúdica e descontraída.

Diversos estudos mostram a importância do uso de simulações no ensino de Física. Arantes et al. (2010, p. 27) ressaltam a importância das simulações computacionais em experimentos, afirmando que "ainda que elas não devam substituir experimentos reais, pesquisas indicam que seu uso combinado à atividade experimental pode tornar mais eficiente o processo de aprendizagem dos alunos".

A primeira etapa do projeto consistiu no desenvolvimento do ambiente Físic@ Virtu@l. Existem diversos repositórios, nacionais e internacionais, disponíveis para livre acesso online, contendo inúmeros objetos de aprendizagem. Dentre todos estes repositórios, pode-se dizer que dois tiveram uma contribuição mais marcante para o ambiente Físic@Virtu@l. Um deles foi o PhET (Physics Education Technology Project), repositório internacional dedicado a produzir objetos de aprendizagem e simulações em diferentes plataformas sobre Física, Química, Biologia e Matemática, que atualmente conta com mais de 360 milhões de simulações. O outro repositório foi o LAPREN $^{2}$, Laboratório de Aprendizagem da Instituição na qual foi desenvolvido o projeto. Os objetos de aprendizagem do LAPREN são desenvolvidos por bolsistas e professores da Universidade, sendo disponibilizados para uso de toda a comunidade acadêmica.

Para o desenvolvimento do ambiente Físic@Virtu@l, primeiramente foi necessária a autorização e liberação do espaço Moodle por parte do setor de educação a distância da Instituição de Ensino Superior (IES) na qual o projeto foi desenvolvido. Após algumas reuniões e esclarecimentos com o coordenador de educação a distância, conseguiu-se a liberação do ambiente virtual, para que os desenvolvedores pudessem adaptar os conteúdos e objetos de aprendizagem, afim de disponibilizá-los futuramente. Para ajudar na comunicação entre os desenvolvedores do ambiente e os estudantes foi criado um Fórum de Informações, no qual são publicados avisos e notícias relacionadas às atividades do Físic@Virtu@l.

No ambiente há duas categorias de objetos de aprendizagem: os provindos do LAPREN e adaptados para o Físic@Virtu@l e os desenvolvidos especificamente para o ambiente. Portanto, a segunda etapa do projeto constituiu-se na adaptação dos objetos de aprendizagem disponíveis no LAPREN, utilizados em oficinas ministradas anteriormente aos estudantes pela professora responsável pelo projeto, e na criação de novos objetos específicos para o ambiente Físic@Virtu@l.O ambiente foi subdividido de tal forma que 
cada aba representasse um objeto de aprendizagem diferente e cada cor identificasse uma área do conhecimento, conforme mostra a figura 1.

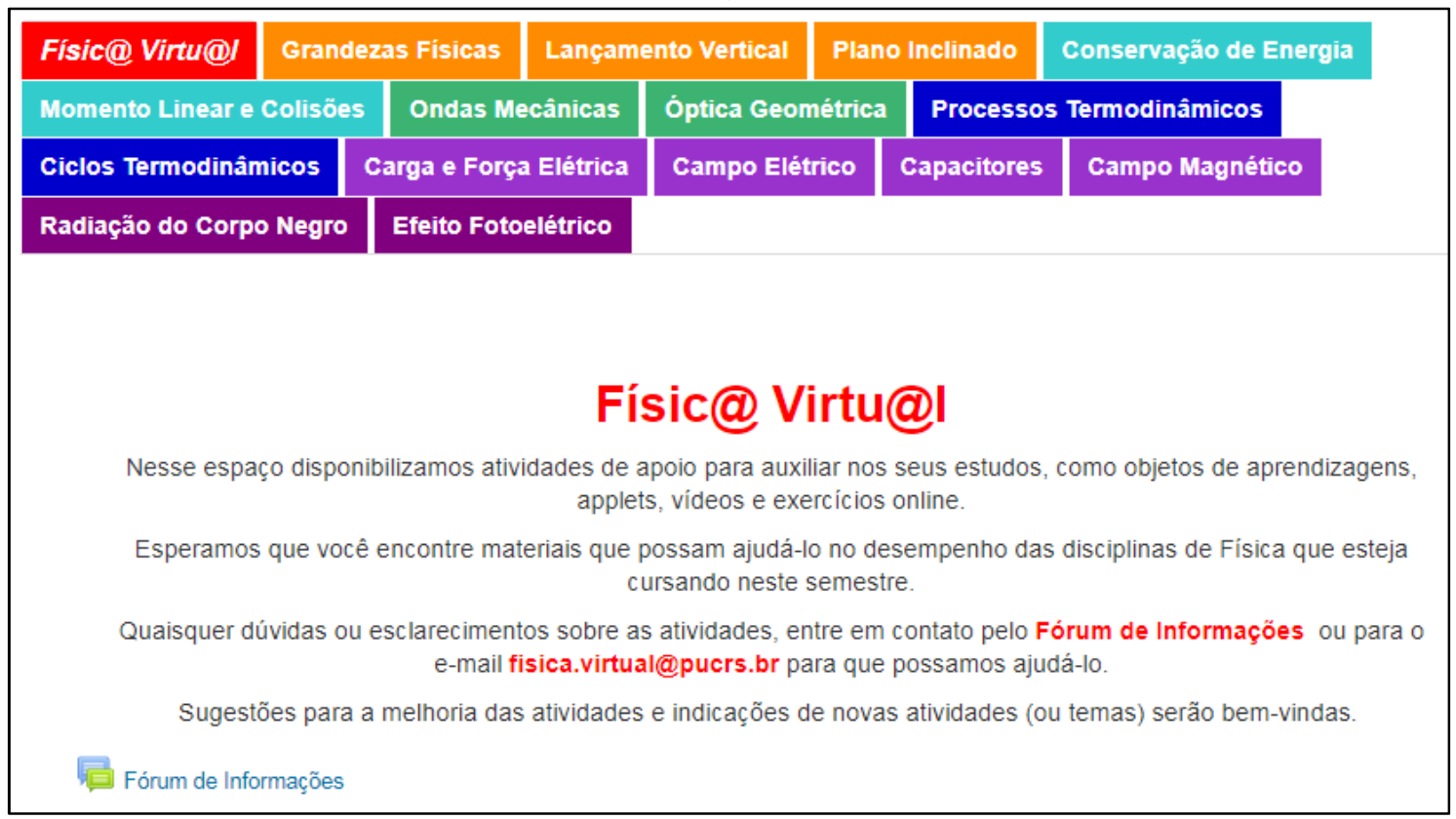

Figura 1 - Interface da página inicial do ambiente Moodle Físic@ Virtu@1

A ideia inicial era utilizar um recurso do Moodle chamado Lição na criação dos objetos de aprendizagem pois, além de ser visualmente mais compacto, este recurso permitia as mesmas funções da interface comum do Moodle com o acréscimo de um banco de questões próprio, em meio aos applets e vídeos. Contudo, isso não foi possível pois houve mudança na versão do Moodle, de 2.2 para 3.0, resultando em erros na Lição. Embora o setor de educação a distância tenha sido contatado e o problema resolvido, optou-se por não utilizar a Lição devido aos erros anteriores, com o receio de que pudessem voltar a ocorrer. Ao invés disso, foi utilizada a interface padrão do Moodle, acrescida de Rótulos, URLs, Arquivos e Questionários.

Os objetos de aprendizagem criados por professores da Física para o LAPREN: Grandezas Físicas, Lançamento Vertical, Plano Inclinado (com e sem atrito), Carga e Força Elétrica, Campo Elétrico, Capacitores e Campo Magnético foram inseridos no ambiente e, diferentemente de outros objetos, apresentam atividades (exercícios, reflexões, testes) dentro de sua animação (Lagreca et al., 2012). A criação dos objetos no ambiente seguiu o mesmo padrão destes, com exercícios e reflexões intercalados de recursos interativos, como applets, vídeos e imagens. Além dos objetos de aprendizagem, em todas as abas há um teste de conhecimentos e uma avaliação do ambiente.

Para abranger as demais áreas do conhecimento das disciplinas de física básica, fez-se necessária a criação de outros objetos de aprendizagem. Entretanto, em contraste com os demais, os objetos criados durante a vigência do projeto não poderiam ser feitos com animação em Flash, devido ao longo tempo necessário para a criação dos mesmos. Ao invés disso, foram escolhidos diversos recursos interativos, como applets, vídeos e imagens. Para reforçar o objetivo de auxiliar a construção e/ou aprimoramento das competências básicas e trazer uma maior dinamicidade ao ambiente, foram formuladas algumas perguntas e exercícios em cada objeto criado, tornando-os mais semelhantes àqueles existentes no LAPREN. 
As primeiras áreas do conhecimento a inspirarem a criação de novos objetos de aprendizagem foram aquelas cujas oficinas mencionadas anteriormente abordavam. Isso deu-se pelo fato de já existir um material de apoio aos quais os alunos poderiam ter acesso, faltando somente a sua transformação para o ambiente virtual para a difusão de tal material.

Alguns objetos de aprendizagem adaptados de oficinas contaram somente com applets retirados de sites de acesso público e exercícios teóricos que envolviam tais applets. Esses objetos diferenciaram-se dos demais, pois possuíam previamente uma série de exercícios teóricos que contribuíram para desenvolver as competências básicas relacionadas à linguagem matemática e à compreensão das equações que expressam os princípios relacionados aos conceitos abordados em disciplinas de física básica.

Em seguida foram criados os demais objetos de aprendizagem, baseando-se nas disciplinas de física básica e seus conteúdos abordados. Esses objetos diferem-se dos demais construídos a partir de oficinas pois, além de menor utilização de exercícios teóricos e matemáticos, contam com vídeos e imagens no auxílio à aprendizagem. Assim como os applets, todo o conteúdo de origem externa é de acesso público.

O objetivo de ambos os tipos de objetos é desafiar o aluno a interagir com os recursos disponibilizados, buscando soluções para as atividades propostas com o auxílio de tais recursos. Isto contribui para dinamizar as atividades e desenvolver a autonomia dos estudantes, que precisam de dados experimentais, mesmo que sendo virtuais, para a realização dos exercícios propostos.

Ao final da criação dos objetos de aprendizagem, fez-se um teste de conhecimentos, a fim de exercitar e verificar o nível de compreensão dos alunos com relação à área do conhecimento abordada pelos referidos objetos. O teste foi desenvolvido utilizando-se o recurso Questionário do Moodle, sendo formuladas algumas questões de múltipla escolha, verdadeiro ou falso, preenchimento de lacunas, entre outras. Os testes contribuíram para os alunos avaliarem a sua compreensão sobre os conteúdos explorados no objeto e, também, para os professores avaliarem as implicações do objeto na aprendizagem dos estudantes.

A partir da conclusão do ambiente Físic@Virtu@l, esperou-se até o início do segundo semestre de 2016 para disponibilizá-lo aos alunos. Ao todo foram 1175 alunos inscritos, abrangendo 14 disciplinas e 59 turmas. A partir da disponibilização do Físic@ Virtu@l, qualquer aluno matriculado em disciplinas que o ambiente abrangia tinha total acesso a todos os conteúdos e objetos de aprendizagem, não se restringindo àqueles referentes à disciplina que o aluno estava cursando. Foi enviado um e-mail aos estudantes matriculados, usando o Fórum de Informações, para explicar o objetivo do ambiente e convidar os alunos a participarem das atividades. Ao longo do semestre o Fórum foi usado para a divulgação dos objetos e incentivo de seu uso.

A quarta etapa consistiu na manutenção do Físic@Virtu@l e na elaboração e criação de novos objetos de aprendizagem, bem como no aperfeiçoamento dos existentes. O processo de manutenção consistiu na constante observação e criação de tabelas de dados provindos dos relatórios do Moodle (número de acesso, notas, frequência de acessos, etc.), com vistas à avaliação do ambiente. Também fazia parte deste processo a manutenção e/ou troca de recursos disponibilizados nos ambientes, bem como o conserto de links e imagens errados e applets defeituosos. Um exemplo de manutenção foi um problema com o recurso Questionário do Moodle. Após testes, verificou-se que em certas ocasiões o recurso não aceitava caracteres que tivessem acentos. Para solucionar esse 
problema foi necessário contato com a coordenadoria de educação a distância, responsável pela manutenção dos recursos do Moodle.

A criação de novos objetos e o melhoramento dos existentes foi necessária para $\mathrm{o}$ aperfeiçoamento do ambiente, a fim de dar continuidade ao trabalho anterior e abordar cada vez mais os conteúdos das disciplinas de física básica.

Como última etapa no desenvolvimento do ambiente no Moodle, foi criado um instrumento de avaliação do ambiente e dos conteúdos abordados, visando aperfeiçoar o ambiente e os objetos de aprendizagem. Como os objetos foram projetados a partir de um padrão, fez-se o mesmo instrumento para todos, usando perguntas que abrangessem de modo geral o funcionamento de cada um. Ao todo, foram dispostos 14 instrumentos de avaliação, um em cada aba do ambiente. A análise dos dados coletados por meio dos instrumentos é apresentada a seguir.

\section{Apresentação dos resultados}

A coleta de dados referentes ao ambiente virtual, tais como número de acessos, objetos acessados e média de notas foi realizada bimestralmente, ou seja, a cada dois meses eram coletados tais dados do relatório gerado pelo Moodle. Isso coincidiu com as datas das duas provas realizadas nas disciplinas, um dado importante para a interpretação dos resultados. A tabela 1 mostra a utilização do ambiente ao longo do segundo semestre letivo de 2016.

Tabela 1 - Número de acessos ao ambiente Físic@ Virtu@1 e aos O.A.

\begin{tabular}{|l|c|c|c|}
\hline Data da coleta de dados & $\mathbf{1 2 / 0 9 / 2 0 1 6}$ & $\mathbf{1 0 / 1 1 / 2 0 1 6}$ & $\mathbf{1 0 / 0 1 / 2 0 1 7}$ \\
\hline $\mathbf{N}^{\mathbf{0}}$ de alunos que acessaram o ambiente & 560 & 600 & 659 \\
\hline $\mathbf{N}^{\mathbf{o}}$ de alunos que acessaram algum O.A. & 177 & 236 & 244 \\
\hline
\end{tabular}

Os dados da tabela evidenciam que do total de 1175 alunos, 47,6\% acessaram o ambiente, sendo que $15 \%$ acessaram algum objeto de aprendizagem no período de um mês após a disponibilização do ambiente. Dois meses após a primeira verificação de utilização, foi realizada a segunda, mostrando que $51,1 \%$ dos alunos matriculados já haviam entrado pelo menos uma vez no ambiente desde a disponibilização e $20 \%$ havia acessando algum objeto de aprendizagem. Na coleta realizada após o término do semestre, verificou-se que $56 \%$ dos alunos haviam acessado o ambiente, com $20,7 \%$ de acessos a algum objeto de aprendizagem.

A partir desses dados podemos supor algumas conclusões. A primeira relacionase com o número total de acessos durante os primeiros meses que, um mês depois da matrícula, já atingia um total de $47,6 \%$ do total de alunos, seja por terem lido o e-mail mandado, para estudar ou por simples curiosidade. Este percentual pode ser considerado satisfatório, levando-se em consideração que o ambiente virtual não era usado nem divulgado em disciplinas nas quais os alunos estavam matriculados, isto é, ele era um ambiente virtual de apoio à aprendizagem independente, não sendo divulgado nem requisitado acesso por nenhum professor das referidas disciplinas. É provável que se os professores utilizassem o Físic@Virtu@l de alguma maneira em suas aulas, o número de acessos fosse maior. Essa afirmação é baseada no fato de que a única divulgação do ambiente Físic@Virtu@l ocorreu no início do semestre. Após isso, nenhum tipo de 
lembrete foi enviado aos que não haviam acessado o ambiente, sendo que somente os estudantes que entrassem no Moodle e procurassem, encontrariam o ambiente. Portanto, se o professor da disciplina os lembrasse da existência, mais alunos poderiam tê-lo conhecido, até aqueles que não acessaram seus e-mails no início do semestre.

Com o passar do tempo, a porcentagem de acessos aumentou de 47,6\% para $51,1 \%$ e $56 \%$ ao final do semestre. Estes dados podem trazer questões passíveis de investigações futuras: os alunos que não acessaram o ambiente, não o fizeram por não terem entrado em nenhum momento em sua conta no Moodle durante o semestre ou por desconhecimento do ambiente e falta de interesse em conhecê-lo?

Com relação aos objetos de aprendizagem, pode-se dizer que os acessos foram mais escassos, sendo usados somente por $15 \%$ dos alunos em seu primeiro mês. Esse número aumentou para $20 \%$ nos dois meses seguintes e, após o término do ano letivo, verificou-se que somente $20,7 \%$ do total de alunos acessou algum objeto de aprendizagem.

Acredita-se que o principal fator para a pouca utilização dos objetos de aprendizagem esteja ligado ao interesse dos alunos pois, embora o ambiente fosse extracurricular, seu objetivo estava anunciado na primeira página: auxiliar os estudos. Se o aluno que entrou no ambiente nem sequer clicou em um link para abrir um objeto de aprendizagem, provavelmente só estava curioso para saber o que era aquele ambiente, não estando necessariamente interessado em aprimorar seus estudos ou testar seus conhecimentos.

A partir dos relatórios retirados do Moodle, foi possível obter o número de acessos a cada objeto de aprendizagem. Os dados foram retirados usando a mesma periodicidade do número de acessos ao Físic@ Virtu@1, citado na seção anterior. A tabela 2 mostra o número de acessos a cada objeto de aprendizagem nos três períodos considerados.

Tabela 2 - Número de acessos a cada objeto de aprendizagem

\begin{tabular}{|l|c|c|c|}
\hline Data da coleta de dados & $\mathbf{1 2 / 0 9 / 2 0 1 6}$ & $\mathbf{1 0 / 1 1 / 2 0 1 6}$ & $\mathbf{1 0 / 0 1 / 2 0 1 7}$ \\
\hline Grandezas Físicas & 42 & 46 & 47 \\
\hline Lançamento Vertical & 17 & 19 & 21 \\
\hline Plano Inclinado Sem Atrito & 03 & 06 & 07 \\
\hline Plano Inclinado Com Atrito & 16 & 18 & 19 \\
\hline Conservação Energia 1 & 06 & 13 & 16 \\
\hline Conservação Energia 2 & 04 & 08 & 08 \\
\hline Momento e Colisões & 02 & 08 & 12 \\
\hline Ondas Mecânicas & 08 & 10 & 10 \\
\hline Óptica Geométrica & 03 & 06 & 06 \\
\hline Processos Termodinâmicos & 18 & 21 & 22 \\
\hline Ciclos Termodinâmicos 1 & 05 & 06 & 08 \\
\hline Ciclos Termodinâmicos 2 & 03 & 03 & 05 \\
\hline Carga e Força Elétrica & 17 & 22 & 24 \\
\hline Capacitores & 08 & 11 & 12 \\
\hline Campo Elétrico & 19 & 21 & 22 \\
\hline Campo Magnético & 06 & 08 & 14 \\
\hline
\end{tabular}

A partir dos dados da tabela 2 podem ser tiradas algumas conclusões. A primeira diz respeito ao objeto mais acessado, Grandezas Físicas. De todos os objetos de 
aprendizagem, pode-se dizer que este seria o mais básico, afinal seu conteúdo é usado em todos os outros. Este objeto trata do conceito de unidades de medida, grandezas escalares e vetoriais, conhecimento básico para um bom aproveitamento de qualquer disciplina de física.

Outra conclusão diz respeito a quais objetos foram mais acessados. Percebeu-se que os objetos mais acessados foram os retirados do LAPREN. Isso pode ocorrer em função de o objeto ser mais sucinto e utilizar recurso de animações que o tornam mais interativo. Com relação ao objeto Processos Termodinâmicos, o número relativamente elevado de acessos pode ser justificado pela grande quantidade de turmas dos cursos de engenharia que cursam disciplinas que abordam tal conteúdo.

Mais uma vez, atenta-se ao fato de que a maior quantidade de visualizações nos objetos deu-se no primeiro mês, reforçando ainda mais a conclusão de que o incentivo ao uso causado pelas notificações no e-mail de cada usuário teve um papel importante.

Com relação aos testes de conhecimentos disponibilizados no Físic@Virtu@l, o Moodle disponibiliza aos organizadores do ambiente um relatório contendo a nota média de cada usuário, a qual foi utilizada para calcular a média de notas do teste de cada objeto de aprendizagem. A tabela 3 apresenta o nível de realização de cada teste (completamente realizado, parcialmente completo ou somente visualizado), bem como a nota média dos estudantes (escala de 0 a 10), na coleta realizada ao término da disciplina.

Tabela 3 - Nível de realização de cada teste e nota média obtida pelos estudantes

\begin{tabular}{|l|c|c|c|c|}
\hline \multicolumn{1}{|c|}{ Teste } & Completo & $\begin{array}{c}\text { Parcialmente } \\
\text { Completo }\end{array}$ & $\begin{array}{c}\text { Somente } \\
\text { visualizado }\end{array}$ & Nota média \\
\hline Grandezas Físicas & 20 & 8 & 9 & 5,36 \\
\hline Lançamento Vertical & 10 & 2 & 2 & 8,4 \\
\hline $\begin{array}{l}\text { Plano Inclinado Com } \\
\text { Atrito }\end{array}$ & 2 & 1 & 0 & 6,58 \\
\hline $\begin{array}{l}\text { Plano Inclinado Sem } \\
\text { Atrito }\end{array}$ & 5 & 1 & 0 & 5,75 \\
\hline Conservação Energia & 4 & 0 & 1 & 7,5 \\
\hline Momento e Colisões & 2 & 1 & 0 & 9,39 \\
\hline Ondas Mecânicas & 0 & 2 & 1 & 4,33 \\
\hline Óptica Geométrica & 0 & 0 & 2 & - \\
\hline $\begin{array}{l}\text { Processos } \\
\text { Termodinâmicos }\end{array}$ & 2 & 0 & 2 & 7,83 \\
\hline Ciclos Termodinâmicos & 0 & 0 & 0 & - \\
\hline Carga e Força Elétrica & 10 & 0 & 1 & 8,85 \\
\hline Capacitores & 3 & 0 & 1 & 9,24 \\
\hline Campo Magnético & 1 & 0 & 3 & 9 \\
\hline Campo Elétrico & 9 & 1 & 5 & 8,63 \\
\hline
\end{tabular}

Como citado anteriormente, um dos pontos importantes a serem destacados é a nota do objeto de aprendizagem Grandezas Físicas. Sendo este o objeto de aprendizagem e o teste de conhecimento mais acessado, parece ter chamado mais a atenção dos alunos, porém a média de notas foi uma das piores, 5,36 de um total de 10 pontos. Isso reforça a necessidade de ferramentas que auxiliem o desenvolvimento das competências básicas, aspecto que motivou o desenvolvimento deste projeto. 
Assim com os objetos, os testes mais acessados foram aqueles que continham objetos de aprendizagem retirados do LAPREN. Conclui-se que, sendo a animação e os recursos utilizados (vídeos, imagens e exercícios) a única diferença entre os objetos criados e os retirados do LAPREN, estes atraem mais a atenção dos alunos devido a sua interatividade e diferença na abordagem do conteúdo.

Em razão do número de estudantes que fizeram os testes ser pequeno em relação ao total de matriculados, não é possível generalizar em quais disciplinas ou conteúdos eles apresentam maior dificuldade. Para realizar generalizações, seria necessária uma amostra maior, o que reforça a necessidade de maior divulgação do ambiente entre os estudantes, para a obtenção de resultados mais expressivos. No entanto, os dados coletados precisam ser levados em consideração, pois mesmo não permitindo uma generalização, nos ajudam a compreender as repercussões do ambiente virtual.

Com relação à avaliação do ambiente Físic@Virtu@l pelos estudantes, o instrumento a ser preenchido era composto de oito questões objetivas. Embora um pequeno percentual de estudantes tenha respondido às questões, a análise das respostas é apresentada a seguir.

Com relação à clareza na apresentação do conteúdo, a maioria dos alunos considerou que foi clara e suficiente para esclarecer as dúvidas. Pelas respostas, provavelmente o objeto de aprendizagem tenha contribuído para a aprendizagem dos alunos que o acessaram.

No que se refere às animações e simulações do objeto de aprendizagem, os estudantes afirmaram que a quantidade foi suficiente e que as mesmas contribuíram para a compreensão do conteúdo. Essa avaliação pode estar relacionada com a constatação apresentada anteriormente de que as simulações retiradas do LAPREN obtiveram um maior número de acessos.

Ao avaliarem os exercícios propostos nos objetos de aprendizagem, os estudantes divergiram. Aproximadamente $60 \%$ concordou com a quantidade de exercícios e os restantes não acharam adequada, mas não justificaram a razão. No entanto, todos concordaram que os comentários apresentados nos exercícios, após a seleção das alternativas, levaram à reflexão sobre a questão proposta. Conclui-se que, apesar de alguns alunos não concordarem com o número de exercícios, eles os ajudaram a refletir sobre as questões abordadas e, somando-se à resposta da pergunta inicial, ajudaram no esclarecimento de suas dúvidas.

Por fim, os estudantes avaliaram que o uso do objeto de aprendizagem foi suficiente para a compreensão do conteúdo. Também afirmaram que com o uso dele sentiram-se motivados a buscarem mais informações para ampliar o estudo sobre o tema abordado, evidenciando-se desta forma que o ambiente cumpriu o papel de auxiliar a aprendizagem dos alunos e também os motivou a ir além, assumindo-se como protagonistas do seu processo de aprendizagem. Este resultado vem ao encontro do que Bulegon (2011) evidenciou em sua tese que os objetos de aprendizagem contribuem para o desenvolvimento da autonomia dos estudantes e para que sintam-se corresponsáveis por sua aprendizagem, definindo a profundidade e o rumo que desejam dar ao estudo de Física.

A avaliação realizada pelos estudantes evidenciou que o ambiente contribuiu para a compreensão de conceitos básicos de física, motivando-os a continuar pesquisando sobre os tópicos abordados. Também foi possível concluir que o ambiente está sendo desenvolvido de forma proveitosa, com exercícios pertinentes, apresentação clara dos 
objetos de aprendizagem e animações e simulações úteis. Além disso, os resultados indicam que, se os alunos forem mais estimulados a entrarem no ambiente e acessarem os objetos, como evidenciado nos primeiros meses de uso do Físic@Virtu@l, a tendência é um acréscimo no número de acessos e participação mais efetiva na avaliação.

\section{Considerações finais}

A necessidade de mudanças na educação básica é um assunto muito debatido nos últimos tempos, tanto no meio acadêmico como em outros. São diversas as opiniões sobre o que está ruim e sobre o que deveria ser feito para qualificar a educação, no entanto são poucas as ações neste sentido. Uma das críticas recorrentes diz respeito à pouca consistência dos conhecimentos construídos ao longo da educação básica, que acarretam dificuldades na formação em nível superior. Tais dificuldades podem causar a reprovação e até mesmo a evasão dos estudantes nos cursos de graduação.

Neste cenário, os objetos de aprendizagem emergem como uma possível estratégia para superar essas dificuldades, servindo de apoio à aprendizagem, ajudando os estudantes na reconstrução de conceitos e no desenvolvimento de competências básicas para um bom aproveitamento das disciplinas a serem cursadas na graduação. Pensando nisso, foi desenvolvido na plataforma Moodle o ambiente virtual Físic@Virtu@l, com o intuito de disponibilizar objetos de aprendizagem, simulações, vídeos e exercícios, auxiliando os alunos que cursam disciplinas de física básica.

É possível concluir que ambientes como o Físic@Virtu@l podem contribuir para a aprendizagem dos alunos, servindo de apoio para superar fragilidades em conceitos básicos e também para desenvolver a autonomia nos estudos. É importante que o acesso a tais ambientes seja estimulado para que mais alunos possam se beneficiar da sua utilização.

Aos professores, a análise dos relatórios disponibilizados pela ferramenta, aliados ao diálogo com os alunos, podem ajudar a compreender melhor a trajetória de aprendizagem dos mesmos. A partir dessa compreensão e da reflexão sobre a própria prática, é possível rever caminhos e traçar novos rumos que permitam qualificar os processos de ensino e de aprendizagem.

\section{Referências}

ARANTES, A. R.; MIRANDA, M. S.; STUDART, N. Objetos de aprendizagem no ensino de física: usando simulações do PhET. A Física na Escola, São Paulo, v.11, n. 1, p. 27-31, abr. 2010.

BULEGON, A. M. Contribuições dos Objetos de Aprendizagem, no Ensino de Física, para o desenvolvimento do Pensamento Crítico e da Aprendizagem Significativa. Porto Alegre: Faculdade de Educação/UFRGS, 2011. 156 p. Tese de Doutorado.

GOMES, G. H.; LOPES, C. M. C.; NIETO, S. S. Cálculo zero: uma experiência pedagógica com calouros nos cursos de engenharia. In: CONGRESSO BRASILEIRO DE ENSINO DE ENGENHARIA, 33., 2005, Campina Grande. Anais. Campina 
Grande: Associação Brasileira de Educação em Engenharia/Universidade Federal de Campina Grande. 2005.

LAGRECA, M. C. B.; MORAES, M. C.; LIMA, V. M. R.; RAYMUNDO, V. P.; GESSINGER, R. M. Estudo do lançamento vertical: uma proposta de ensino por meio de um objeto de aprendizagem. Caderno Brasileiro de Ensino de Física,

Florianópolis. v. 29, n. especial 1, p. 543-561, set. 2012.

NICOLA, L. B.; RODRIGUES, A. P. Objetos de Aprendizagem como

Potencializadores no Estudo da Biologia. Renote, Porto Alegre, v. 9, n. 1, p.1-10, jul. 2011.

QUARTIERI, M. T.; BORRAGINI, E. F.; DICK, A. P. Superação de dificuldades no início dos cursos de engenharia: introdução ao estudo de física e matemática. In:

In: CONGRESSO BRASILEIRO DE ENSINO DE ENGENHARIA, 40., 2012, Belém. Anais. Belém: Associação Brasileira de Educação em Engenharia/Universidade Federal do Pará. 2012.

SABBATINI, M. Reflexões críticas sobre o conceito de objeto de aprendizagem aplicado ao ensino de Ciências e Matemática. Em Teia - Revista de Educação

Matemática e Tecnológica Iberoamericana, Recife, v. 3, n. 3, p.1-36, 2012. SOBRINHO, M. C.; CARDOSO, P. C. F.; FAVERO, E. L. Objetos de Aprendizagem no Ensino de Inglês. Renote, Porto Alegre, v.4, n.2, p.1-9, dez. 2006.

\section{Notas}

${ }^{1}$ https://phet.colorado.edu/pt_BR/simulations/category/physics

${ }^{2}$ https://lapren.pucrs.br/ 